\title{
Linx
}

LinX Revue des linguistes de l'université Paris X Nanterre

$54 \mid 2006$

La cause : approche pluridisciplinaire

\section{Causalité en grec moderne : réflexions sur la prise en charge énonciative et la modalité}

Eleni Valma

\section{OpenEdition}

\section{Journals}

Édition électronique

URL : http://journals.openedition.org/linx/533

DOI : $10.4000 /$ linx.533

ISSN : 2118-9692

Éditeur

Presses universitaires de Paris Nanterre

\section{Édition imprimée}

Date de publication : 1 juin 2006

Pagination : 181-207

ISSN : 0246-8743

\section{Référence électronique}

Eleni Valma, « Causalité en grec moderne : réflexions sur la prise en charge énonciative et la

modalité », Linx [En ligne], 54 | 2006, mis en ligne le 01 août 2007, consulté le 01 mai 2019. URL:

http://journals.openedition.org/linx/533; DOI : 10.4000/linx.533 


\title{
Causalité en grec moderne : réflexions sur la prise en charge énonciative et la modalité*
}

\author{
Eleni Valma, LACIYO-UME 7107 \\ Centre d'études balkaniques, INALCO \\ Université Paris $X$ - Nanterre
}

\section{Introduction - Cadre théorique}

L'expression linguistique de la notion de «causalité » en grec moderne n'a pas fait l'objet de nombreuses études. Les premiers articles publiés sur le sujet ont longtemps suggéré une description des connecteurs causaux en se fondant notamment sur les trois niveaux d'analyse proposés par Sweetser [1990/1998: 811․ Si les niveaux que distingue Sweetser dans le chapitre 4 de son ouvrage décrivent de façon sans doute convaincante l'emploi du connecteur anglais because (fr. parce que, car), la réalité linguistique du grec moderne impose d'emblée, nous semble-t-il, une problématique plus complexe susceptible de rendre compte de la diversité des moyens que cette langue possède ${ }^{2}$. D'ailleurs, Koutoupi-Kitis [à paraître, p. 7] a récemment renoncé à l'analyse

\footnotetext{
* Notre gratitude va à Isabelle Bril, Jean Lallot et Sophie Vassilaki pour avoir accepté de relire notre travail et pour leurs commentaires précieux.

1 «Causal conjunction in the speech-act domain indicates causal explanation of the speech act being performed, while in the epistemic domain a causal conjunction will mark the cause of a belief or a conclusion, and in the content domain it will mark real-word causality of an event. »

${ }^{2}$ Galiotou [1999: 8, 174] a adopté la distinction entre «information causale explicite» et « information causale implicite» présentée dans Nazarenko [1994, Compréhension de la causalité du
} 
de Sweetser. Elle a eu recours à une distinction entre objectivité et subjectivité ${ }^{3}$ [Koutoupi-Kitis, à paraître, p. 26] qui tient compte du rôle des connaissances extralinguistiques (« standing background knowledge and beliefs ») ou contextuelles (" current mutual contextual assumptions»). Koutoupi-Kitis fait également appel à l'analyse étymologique des connecteurs causaux afin de mettre en évidence leurs traits distinctifs. Nous avons déjà évoqué dans Valma $(2004$ : 562 ; 2005 : 89) la nécessité de passer par cette voie pour identifier les divers emplois ${ }^{4}$.

Dans cet article, nous proposons une analyse sémantique de trois connecteurs

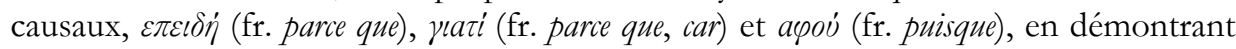
que leur trait distinctif réside dans la notion d'engagement et de désengagement de l'énonciateur par rapport à la relation existant entre deux faits, dont l'un est la cause et l'autre l'effet. Cette hypothèse, de par sa nature même, implique des éclaircissements théoriques sans pour autant prétendre proposer de nouvelles perspectives théoriques.

Une phrase se compose d'un modus et d'un dictum, terminologie héritée du Moyen Age et longuement exposée par Bally [1932/1965 : 36]. Si le dictum renvoie à ce qui est dit, le modus est étroitement lié à la modalité, à l'attitude du sujet vis-à-vis d'un contenu propositionnel. En articulant une phrase, on effectue un acte de langage (acte locutoire, un acte illocutoire et un acte perlocutoire). L'acte illocutoire connait trois formes (affirmative, interrogative et impérative) et possède des valeurs comme l'assertion, la question, l'ordre, la promesse, etc. L'assertion consiste à transmettre un contenu propositionnel sur le mode de dire $e^{5}$ et ne doit pas se confondre avec la notion de vérité. Nous considérons qu'une construction attribue à l'énoncé sa valeur tout en se réalisant dans une situation énonciative précise. Ainsi, on construit un univers de validation [Culioli, 1990: 43] et on fait apparaître un énonciateur qui s'engage sur l'existence d'un état de choses. Il s'agit alors d'une évaluation d'un contenu propositionnel sans se référer à l'opposition objectivité-subjectivité. Cette évaluation comprend le vrai et le faux et se lie ainsi à la modalité. Si la littérature propose plusieurs types de modalité [Palmer, 2001 : 22], la modalité aléthique et la modalité épistémique [Bybee \& Fleishman, 1995: 6] attirent particulièrement notre attention, car la modalité aléthique implique directement la notion du certain et du nécessaire alors que la modalité épistémique exprime l'engagement de l'énonciateur par rapport au contenu

langage naturel, thèse de doctorat, Université Paris XIII, Paris] : la première est exprimée par des marqueurs causaux de la langue qui sont présents dans le texte, tandis que la seconde est déduite du réseau de contraintes temporelles au moyen du mécanisme d'inférence. En suivant le modèle d'Asher [1993, Reference to abstract objects in discourse, Editions Kluwer Academic Publishers, Dordrecht], Galiotou fait intervenir la notion de temps pour hiérarchiser la relation établie entre deux faits.

3 «I suggested that a division of labour between an objective and a subjective world as a basis for an initial account of the function of causal connectives might be a better solution than Sweetser's domains. »

${ }^{4}$ Dans notre première étude du phénomène de causalité [1999: 42, 43], nous avions déjà signalé l'importance de l'analyse étymologique et le type de cause véhiculée par $\varepsilon \pi \varepsilon \iota \delta \dot{~(f r . ~ p a r c e ~ q u e) ~ e t ~ \gamma ı a \tau i ~}$ (fr. parce que, car). A l'époque, nous avions opté pour la distinction entre «causalité objective» et « causalité subjective ». Les lectures qui ont suivi ce travail ont clairement montré les faiblesses d'une telle terminologie que nous avons choisi d'écarter au profit de théories plus appropriées (cf. modalité).

${ }^{5}$ Le simple acte d'énonciation renvoie à la notion de dire et son contenu au dit [Ducrot, $\left.1984: 177\right]$. 
de son énoncé. L'engagement de l'énonciateur se traduit par une prise en charge énonciative, à savoir un énonciateur se portant garant sur le contenu propositionnel ou sur la relation établie entre deux propositions.

Nous devons clairement identifier deux «personnages» du discours, l'énonciateur et le locuteur ${ }^{6}$ :

L'énonciateur est celui qui prend matériellement en charge l'énoncé, tandis que le locuteur dépend de l'énonciateur et il est censé proférer un acte d'énonciation qu'il soit réel ou non. [Desclés \& Guentchéva, 2000 : 84]

Outre la prise en charge énonciative lors d'une relation causale, nous nous situons dans une perspective communicative où l'énonciateur souhaite avant tout informer le co-énonciateur de quelque chose (intention informative dans Sperber \& Wilson, 1986/1989: 51). Afin qu'il y ait communication, énonciateur et coénonciateur évaluent le contenu propositionnel, apprécient donc la pertinence de la relation établie entre deux propositions.

Cette relation se base souvent sur l'observation des faits et le raisonnement. Selon Peirce [1878, cité dans Desclés \& Guentchéva, 2001 : 105-106], les schémas inférentiels sont facilement répérables grâce à des propositions organisées en règle, cas (ou prémisses) et résultat. Le cas est l'état antérieur, alors que le résultat représente l'effet. Peirce distingue trois raisonnements: l'induction', la déduction' et l'abduction ${ }^{10}$. Bien qu'ils restent des raisonnements réservés principalement aux sciences dures - nous pouvons nous en servir dans une étude linguistique pour expliquer l'inférence traduisant une démarche d'explication des faits. Dans le raisonnement déductif, la combinaison de la règle et du cas observé autorise la conclusion nécessaire du résultat. Le raisonnement abductif diffère de la déduction en ceci : c'est l'observation du résultat, en passant par la règle, qui conduit au cas. Ce dernier est qualifié de plausible ${ }^{11}$.

\footnotetext{
${ }^{6}$ Distinction faite par Ducrot [1984 : 193, 204] dans le cadre de la théorie de polyphonie.

${ }^{7}$ Nous faisons ici la distinction entre la règle et la loi. Une loi relève d'un savoir commun et partagé par tous les utilisateurs tandis qu'une règle relève d'un savoir plus local et moins partagé. [Desclés \& Guentchéva, $2001: 108$, note 10]

${ }^{8}$ Règle : cette bille vient de ce sac. Cas : cette bille est rouge. Résultat : les billes dans ce sac sont rouges. [exemple de Fitzhugh, 2005, «Les bases philosophiques de l'inférence phylogénétique - une vue d'ensemble », Biosystema 24, p.83-104]

${ }^{9}$ Règle : les billes dans ce sac sont rouges. Cas : cette bille vient de ce sac. Résultat : cette bille est rouge. [exemple de Fitzhugh, idem]

${ }^{10}$ Règle : les billes dans ce sac sont rouges. Cas : cette bille est rouge. Résultat : cette bille vient de ce sac. [exemple de Fitzhugh, idem]

11 Desclés \& Guentchéva [2001 : 105-106] affirment « une proposition plausible n’est pas le résultat d'un jugement qui évaluerait son degré de certitude, mais elle résulte d'un raisonnement qui conduit à un résultat qui est choisi, avec une incertitude, parmi d'autres propositions possibles.»
} 


\section{E $\pi \varepsilon \iota \delta \dot{\eta}:$ la cause}

Dans ce paragraphe, nous essaierons de démontrer que le connecteur $\varepsilon \pi \varepsilon i \delta \dot{\eta}$ (fr. parce que) introduit une cause, un premier fait $\left(\mathrm{Sit}_{1}\right)$ susceptible de déclencher un deuxième $\left(\mathrm{Sit}_{2}\right)$. Le rôle de l'énonciateur se résume à l'énonciation des faits; il s'agit alors d'une assertion simple ou collective ${ }^{12}$. Parce que $\varepsilon \pi \varepsilon \varepsilon \delta \dot{\eta}$ apparait dans un type de constructions précis - que nous présenterons plus loin, la cause qu'il introduit s'interprète comme certaine.

Le connecteur $\varepsilon \pi \varepsilon \varepsilon \delta \eta \dot{~ e s t ~ d i r e c t e m e n t ~ i s s u ~ d u ~ g r e c ~ a n c i e n ~[A n d r i o t i s, ~ 1951 / 1995] ~ o u ̀ ~}$ il véhiculait deux notions, premièrement celle de temps (antériorité) et deuxièmement celle de cause [Liddell \& Scott, 1843/1997].

La proposition subordonnée admet deux positions syntaxiques ${ }^{13}$, l'antéposition et la post-position à la principale, et introduit la thématisation ${ }^{14}$ de la cause :

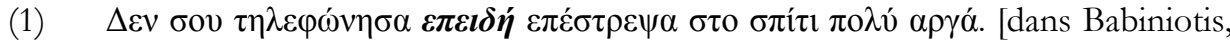
1998]

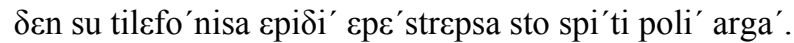

(a) «Je ne t’ai pas appelé parce que je suis rentrée très tard. »

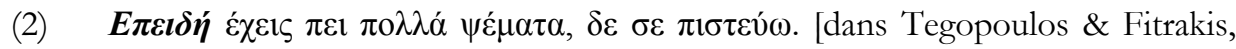
1997]

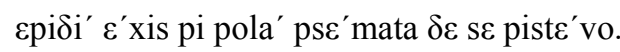

(a) «Parce que tu as déjà beaucoup menti, je ne te crois pas.»

Il faut souligner que, dans l'emploi de ce connecteur, la postposition n'est pas une «raison de dire» (cf. note 5) et l'antéposition n'introduit pas une information ancienne et connue par le co-énonciateur (contra Babiniotis \& Clairis, 2001 : 97, \$107).

Dans les premières études linguistiques, on a voulu trouver le trait distinctif de ce connecteur, par opposition aux autres connecteurs causaux du grec moderne dans son incapacité à se combiner avec deux actes de parole différents [Koutoupi-Kitis, 1994 : 309, 313 ; Koutoupi-Kitis, 1996 : 433] :

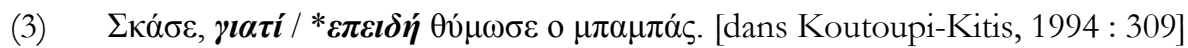

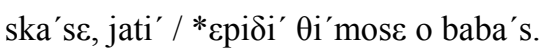

(a) «Tais-toi, parce que papa s'est énervé. »

\footnotetext{
12 Desclés \& Guentchéva [2000 : 91-92] définissent l'assertion collective comme l'affirmation qui appartient au savoir collectif. «C'est donc l'énonciation d'une assertion assumée par la communauté entière à laquelle se joint l'énonciateur.»

13 Dues à l'analyse étymologique de ce connecteur. Pour le mode de la subordonnée, cf. Holton et al., 1997 : 441, \$5.3.11.

${ }^{14}$ La thématisation et la focalisation sont des opérations énonciatives, se manifestant également à travers la syntaxe, qui sont susceptibles de hiérarchiser les informations données dans une phrase.
} 
Quelques années plus tard, Kalokerinos [1999: 144-145] revendiquait la grammaticalité de cette construction. Il faudrait, sans doute, interpréter cette combinaison pour dégager des propriétés non seulement syntaxiques mais également sémantiques de ce connecteur. Nous avons plus haut affirmé que $\varepsilon \pi \varepsilon \varepsilon \delta \eta \eta$ introduit une cause certaine (modalité aléthique) et nous pensons que l'observation de trois phénomènes pourrait renforcer cette hypothèse. Il s'agit de la combinaison de deux actes de parole, du rôle de la négation et des adverbes. Pour ces derniers, nous prêterons attention à la place et au sémantisme d'adverbes combinés avec $\varepsilon \pi \varepsilon \varepsilon \delta \dot{\eta}$.

Dans l'exemple (4), la subordonnée est une proposition interrogative

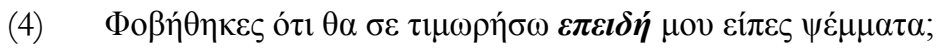

fovi' $\theta i k \varepsilon s$ o'ti $\theta$ a se timori'so $\varepsilon$ pi $\delta i^{\prime}$ mu i'pes pse'mata?

(a) "As-tu eu peur que je te punisse parce que tu m'as menti ? "

La question ne porte pas sur le contenu de la subordonnée car mentir est présenté comme certain. En revanche, on cherche à savoir si cette cause a donné naissance à l'état psychologique avoir peur ou si cet état est dû à autre chose. La négation se comporte de la même façon. En (5) le contenu de la subordonnée n'est pas nié ; mais il est présenté comme l'une des causes susceptibles de produire l'effet décrit dans la principale :

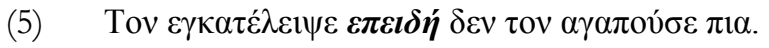

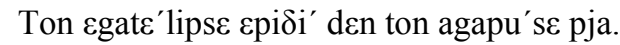

(a) «Elle l'a abandonné parce qu'elle ne l'aimait plus.»

Ainsi, nous voyons qu'une proposition négative ou interrogative ne change pas la nature du contenu de la subordonnée (celui-ci est toujours présenté comme certain); en revanche, on effectue une focalisation et non plus une thématisation comme en (1) et (2). En effet, la combinaison avec deux actes de parole différents (sauf pour le cas de l'ordre, cf. ex. 23) focalise l'attention sur une cause sans exclure la possibilité d'en avoir d'autres qui agissent parallèlement.

L'emploi d'un adverbe souligne, lui aussi, le caractère certain de la subordonnée. Voyons l'exemple (6) qui présente un intérêt particulier du fait du sémantisme de l'adverbe, mais également des valeurs aspecto-temporelles de la subordonnée :

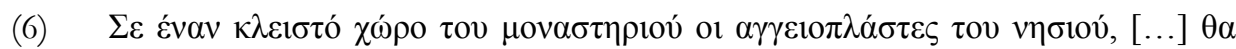

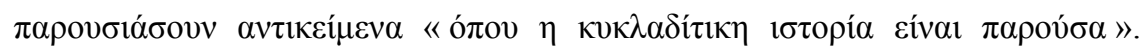

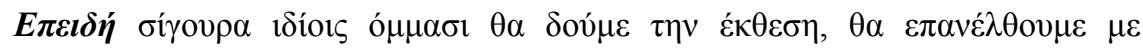

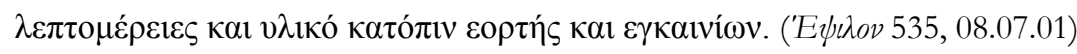

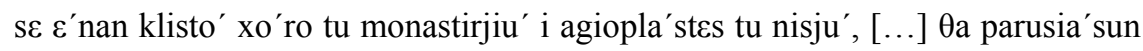

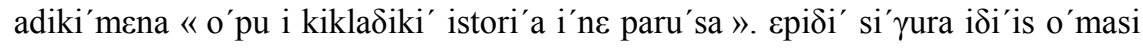

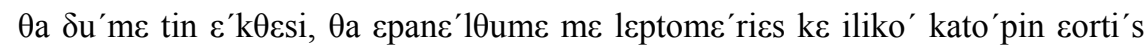
ke Egeni'on. 
(a) «Dans les locaux du monastère, les potiers de l'île, [...] exposeront des objets représentants de l'art cycladique. Et puisque à coup sûr nous visiterons l'exposition de nos propres yeux, nous reviendrons en détails sur le sujet juste après le vernissage. » (Epsilon 535, 08.07.01)

Commençons par l'adverbe oíjov@a (fr. à coup sûr, sûrement, certainement) ; à cause de sa position syntaxique, il porte sur la subordonnée. Si $\varepsilon \pi \varepsilon \iota \delta \eta ்$ introduit le certain, la présence de l'adverbe ó'yovoa (fr. sûrement) ne peut que renforcer cette certitude. Une

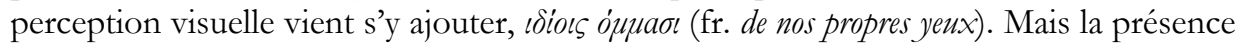
d'un futur dans la subordonnée pourrait remettre en question cette certitude. Car on sait que le futur introduit l'incertain puisqu'il se réfère à une situation à venir ${ }^{15}$. Pour expliquer le futur en (6), nous devons emprunter quelques éléments d'analysés proposés dans [Desclés, 1998-1999] pour le futur proche du français ${ }^{16}$. Le futur de (6) renvoie à un fait à venir, non encore réalisé mais quasi-certain, excluant toute possibilité de ne pas visiter l'exposition, comme le souligne la présence du connecteur $\varepsilon \pi \varepsilon \delta \delta \dot{\eta}$. Pour l'exemple (7), où apparait l'expression figée « $\varkappa_{l} \varepsilon \pi \varepsilon \varepsilon \delta \dot{\eta} ;$; ", nous suivons la même analyse :

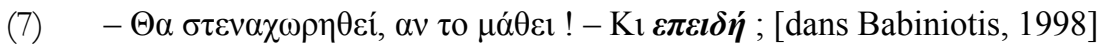

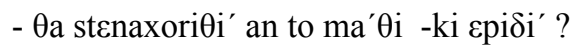

(a) «- Il sera triste, s'il l'apprend ! - Et alors ?» (litt. Et parce que ?)

La tristesse d'une tierce personne est certaine (paraphrasée par «il sera certainement triste ») et on s'interroge uniquement sur les conséquences éventuelles de cette tristesse ; nous proposons donc de le traduire par « et alors ».

Mais ce connecteur, pourquoi présente-t-il le contenu de la subordonnée comme certain malgré la négation, l'interrogation ou encore le futur ? D’après nous, il faudrait chercher dans son emploi temporel du grec ancien. Des études typologiques [Hopper \& Traugott, 1993] ont montré l'évolution notionnelle d'un morphème et proposent des cartes cognitives liant les notions entre elles [Kortmann, $1996: 210]$. Notamment, les travaux de Schwyzer [Griechische Grammatik, 1939: 659, cité dans Koutoupi-Kitis, 1996] pour le cas du grec et les travaux de Traugott \& König [1991 : 190, 195, 197] mentionnent qu'une grande partie des connecteurs causaux a effectivement une origine temporelle. Mais nous souhaitons aller plus loin. Ainsi, nous dirons que les connecteurs causaux anciennement temporels (cf. $\varepsilon \pi \varepsilon i \delta \eta$ ) et les connecteurs temporels à emploi causal (cf. connecteur polysémique a introduisent une cause certaine. A l'origine de ce type de cause se trouve le fait que ces connnecteurs ont introduit ou introduisent encore l'antériorité ou la concomitance.

Il résulte de l'analyse que nous proposons que $\varepsilon \pi \varepsilon l \delta \eta \eta$ introduit une modalité aléthique où la cause est présentée comme certaine; elle est également vraie lorsqu'on utilise un temps du passé ou un présent dans la subordonnée puisque le fait décrit a été constaté ou est constaté en $\mathrm{T}_{0}$ (moment de l'énonciation). Ainsi, le rôle de

\footnotetext{
${ }^{15}$ Le futur du grec moderne est fortement modal surtout lorsque la forme verbale introduit l'aspect inaccompli.

${ }^{16}$ Le futur proche morphologique n'existe pas en grec moderne.
} 
l'énonciateur se résume à une simple assertion susceptible de devenir une assertion générale quand le contenu de la subordonnée a une valeur générique. Tel est le cas de l'exemple suivant

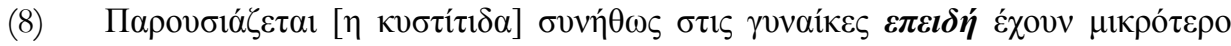

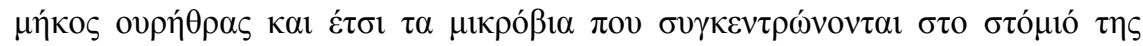

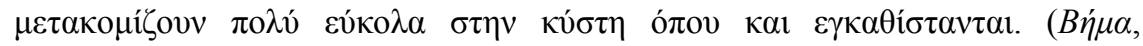

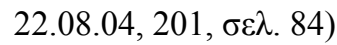

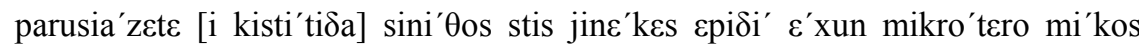
uri' $\theta$ ras ki $\varepsilon$ 'tsi ta mikro'via pu sugedro'nod $\varepsilon$ sto sto'mio' tis metakomi'zun poli' $\varepsilon$ 'fkola stin ki'sti o'pu ke \&ga $\theta i^{\prime}$ stade.

(a) «Elle [la cystite] se manifeste d'ordinaire chez les femmes parce que leur urètre est moins long, ainsi les microbes se trouvant à son orifice migrent facilement vers la vessie où ils s'installent. »(Vima, 22.08.04, 201, p. 84)

Le connecteur $\varepsilon \pi \varepsilon \delta \delta \dot{~ d o i t ~ s o n ~ p o u v o i r ~ e x p l i c a t i f ~ e t ~ l e ~ s e n t i m e n t ~ d ' e ́ v i d e n c e ~ a ̀ ~}$ cette valeur générale ${ }^{17}$. Il est étroitement lié à la compréhension de la relation existant entre deux faits. Cette évidence peut se baser sur l'autorité personnelle de l'énonciateur (son statut, ses connaissances, ...) ou sur une loi. Dans nos travaux antérieurs [2004c], nous avons montré qu'on rencontre $\varepsilon \pi \varepsilon \varepsilon \delta \dot{\eta}$ dans un certain type de raisonnement, à savoir dans la déduction (inférence par modus ponens) ; si les prémisses sont certaines, les conclusions le sont également.

\section{3. $\Gamma \propto \alpha \tau i$ : la cause, la concession et la coordination}

Dans ce paragraphe, nous analyserons quelques aspects du connecteur yrazi (fr. parce que, car) présentant une certaine complexité. Nous avançons l'hypothèse qu'il introduit une cause plausible tout en faisant apparaitre un énonciateur qui se porte garant non seulement du contenu propositionnel de la subordonnée mais également de la relation établie. Parallèlement, nous serons amenée à examiner un autre emploi de ce connecteur, comme conjonction d'opposition.

Si nous observons l'exemple (9), nous constatons que yıati à deux emplois distincts qui ont deux étymologies différentes :

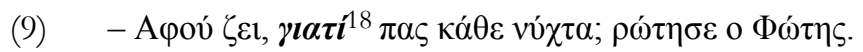

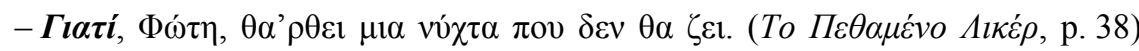
-afu' zi, jati' pas ka' $\theta \varepsilon$ ni'xta? ro'tis $\varepsilon$ Fo'tis. -jati', Fo'ti, $\theta$ a r $\theta$ i mja ni'xta pu $\delta \varepsilon n \theta$ a zi.

${ }^{17}$ Dans un discours explicatif [Borel, 1981 : 9], l'énonciateur se fait témoin des événements qu'il constate et décrit, des structures dans lesquelles entrent ces événements, des actions qu'il subit, des trasformations dont ces événements sont le siège et dont il reproduit le fil.

${ }^{18}$ Kalokerinos [1999: 149] note que l'interrogatif ytazi peut admettre une réponse causale ou téléonomique. 
(a) - Puisqu'elle est vivante, pourquoi y vas-tu chaque nuit? demanda Photis. - Parce qu'une nuit viendra où elle ne vivra plus. Voilà pourquoi.» (La liqueur morte, p. 44)

En tant que particule interrogative, ce connecteur s'analyse en $\delta i a ́+\pi i$ (expression figée en grec ancien, fr. pourquoi ?) ; en tant que connecteur, il introduit une subordonnée causale et s'analyse en $\gamma \iota a+o^{\prime} \iota^{19}$. L'élément commun de ces deux étymologies est la préposition $\delta \iota \dot{~(e n ~ g r e c ~ a n c i e n) ~ e t ~} \gamma \iota a$ (en grec moderne) équivalant à la préposition française pour. La particule anaphorique $\dot{o} \iota$ (fr. que) qui suit, implique la position syntaxique de la subordonnée causale : elle est obligatoirement postposée. Cette post-position introduit une raison de dire. La subordonnée postposée justifie l'énonciation et le contenu propositionnel de la principale qui précède.

A première vue, il semblerait que le connecteur yiati soit réservé à une cause certaine et constatée au même titre que le connecteur $\varepsilon \pi \varepsilon i \delta \eta ்$; cette remarque a amené certains linguistes [Arabatsidou, 1997: 56-67] à considérer les deux connecteurs comme équivalents, au moins sur le plan sémantique :

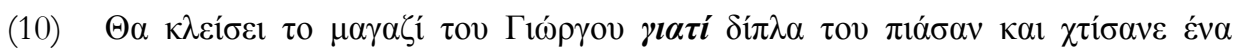

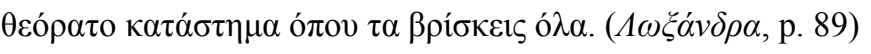

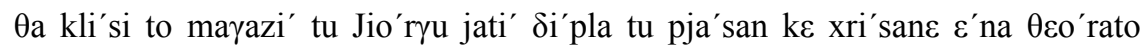
kata'stima o'pu ta vri'skis ola.

(a) «La boutique de Yiorgos allait fermer car un immense magasin où l'on trouvait de tout s'était construit à côté. » (Loxandra, p.86)

Néanmoins, l'analyse de rıazi et la comparaison avec $\varepsilon \pi \varepsilon i \delta \dot{r}$ révèlent des différences importantes entre ces connecteurs.

Dans le paragraphe consacré à $\varepsilon \pi \varepsilon \varepsilon \delta \eta \dot{\eta}$, nous avons abordé le rôle syntaxique et sémantique de l'adverbe accompagnant le connecteur et montré que l'adverbe renforce le caractère certain de la cause marquée par $\varepsilon \pi \varepsilon \delta \delta \eta$. Mais l'adverbe ne se comporte pas de la même façon avec le connecteur yıati. Analysons l'exemple suivant :

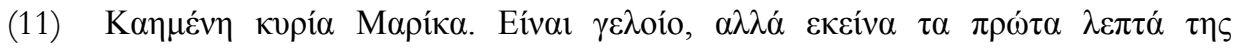

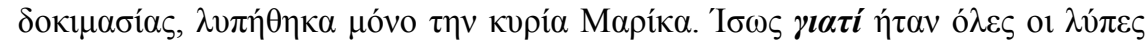

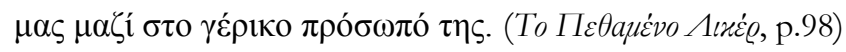

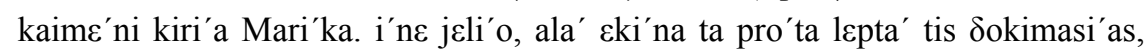
lipi' $\theta i k a$ mo'no tin kiri'a Mari'ka. i'sos jati' i'tan o'les i li'pes mas mazi' sto je'riko pro'sopo' tis.

(a) «Pauvre Kyria Marika. C'est dérisoire, mais en ces premiers instants d'épreuve j'eus seulement pitié de Kyria Marika. Sans doute parce que toutes nos peines se lisaient sur son visage usé. » (La liqueur morte, p. 125)

\footnotetext{
${ }^{19}$ Nous signalons également la variante $\delta \iota \dot{\tau} \iota$ que nous n'examinons pas dans cet article.
} 
En (11), la subordonnée qui suit le verbe $\lambda v \pi \dot{\theta} \theta \eta \varkappa a$ (fr. avoir pitiè) justifie ce sentiment de tristesse. L'adverbe i $\sigma \omega \varsigma$ (fr. peut-être), portant sur la subordonnée, présente son contenu comme plausible et cela est renforcé par yıatí.

Dans l'exemple suivant, yıati justifie l'énonciation d'une situation tout en faisant apparaître l'énonciateur :

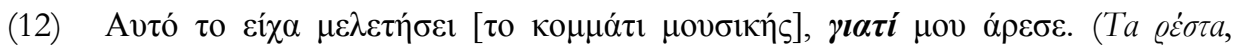
p. 109)

afto' to i'xa meleti'si [to koma'ti musiki's], jati' mu a'rese.

(a) «Celui-là, oui, je l'avais travaillé [ce morceau de musique], il me plaisait. » (La petite monnaie, p. 121) [schéma asynthète pour la traduction française]

Litt. Celui-ci je l'avais étudié [le morceau de musique] car il me plaisait.

Le pronom $\mu o v$ (fr. me), en se combinant avec le connecteur yıati et le verbe

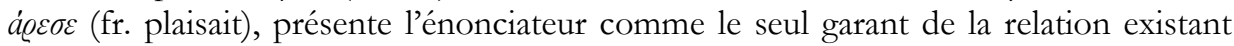
entre deux faits travailler et plaire. Le contenu de la subordonnée n'est pas vérifiable ; on est obligé de croire l'énonciateur sur parole.

Ce connecteur est également employé quand l'énonciateur veut reprendre un terme précédent dans le contexte. Comme le montre (13), yıati est utilisé pour introduire tardivement, comme dans un repentir, une information qui aurait dû être donnée plus tôt :

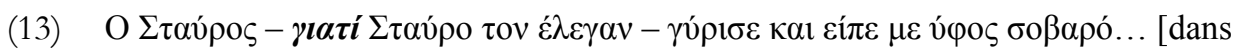
Kalokerinos, 1999 : 145 ; l'auteur y voit un acte illocutoire]

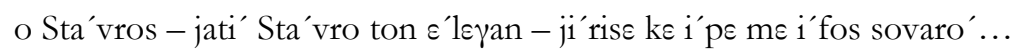

(a) «Stavros - parce qu'il s'appelait Stavros - s'est retourné et nous a dit gravement...»

Nous constatons que - dans ce type d'emploi - le connecteur causal introduit un fait qui est certain et vérifiable à tout moment. Le contenu de la subordonnée peut se situer sur le plan discursif sans qu'il soit indispensable pour la compréhension de la relation entre deux faits :

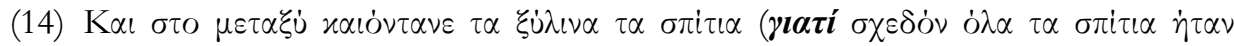

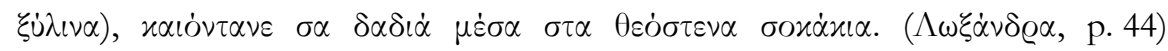

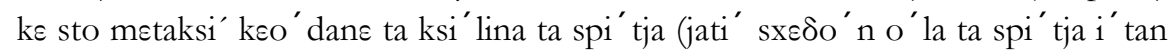
ksi' lina), keo' dan $\varepsilon$ sa $\delta a \delta j a ' m \varepsilon$ 'sa sta $\theta \varepsilon o$ 'stena soka' kja.

(a) «Et au fur et à mesure que les flammes se propageaient, et elles se propageaient vite car presque toutes les maisons étaient en bois, la confusion, le vacarme augmentaient. » (Loxandra, p. 42-43)

Litt. Et en même temps les maisons en bois (car presque toutes les maisons étaient en bois) brûlaient, elles brûlaient comme des torches dans les ruelles trop étroites. 
Ainsi est-il souvent donné en incise. Certaines stratégies publicitaires utilisent ce connecteur avec une fonction de pseudo-explication :

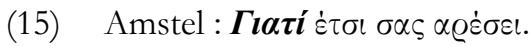

Amstel : jati' $\varepsilon$ 'tsi sas are'si.

(a) «Amstel : parce que c'est comme ça qu'elle vous plait. »

En effet, la proposition introduite par le connecteur causal n'explique rien au choix du consommateur. La relation est injustifiée et présentée de façon dogmatique. Ce même dogmatisme (ou encore refus d'apporter une explication éclaircissant la relation entre deux faits) se manifeste clairement dans l'emploi figé du connecteur. En

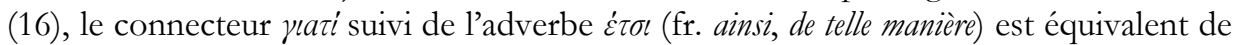
«parce que - point final» :

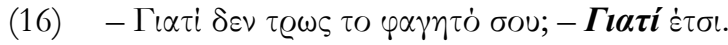

- jati' $\delta \varepsilon n$ tros to fayito' su ? - jati' $\varepsilon$ 'tsi.

(a) «- Pourquoi tu ne manges pas ton repas? - Comme ça.»

Ce connecteur marque donc un fort engagement de l'énonciateur par raport à un fait qui, jusqu'à maintenant, est donné comme incertain. Cela classe yıatí parmi les marqueurs de modalité épistémique. On a certainement besoin d'un énonciateurgarant car, comme le montre (17), on établit souvent une relation à caractère fortement restreint :

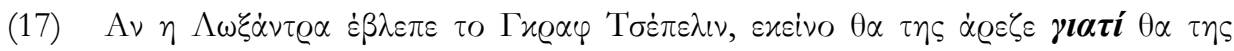

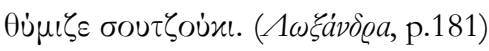

an i Loksa' dra $\varepsilon^{\prime}$ vlepe to Graf Tsepelin, $\varepsilon k i$ ' no $\theta a$ tis a'reze jati' $\theta$ a tis $\theta{ }^{\prime}{ }^{\prime}$ mize sutzu'ki.

(a) «Si elle avait vu le Graf Zeppelin, elle s'en serait fort réjouie car il lui aurait fait penser à une saucisse. » (Loxandra, p.180)

On a des difficultés à généraliser une image associant le Graf Zeppelin à une saucisse ; mais on comprend cette association et la réjouissance qu'elle provoque chez Loxandra si on pense qu'elle est extrêmement gourmande (information donnée dans le contexte).

Cette portée limitée de la relation entre deux contenus aboutit parfois à un raisonnement paradoxal. Le contexte est alors indispensable à l'interprétation de l'énoncé. Nous constatons alors une grande perturbation par rapport à une loi, ce qui entraîne un effort de traitement de l'information considérable de la part du coénonciateur [Sperber \& Wilson, 1986/1989 : 319]. Citons l'exemple :

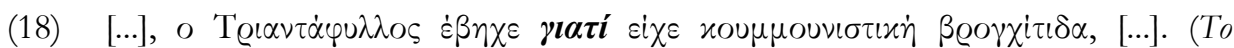

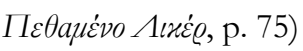

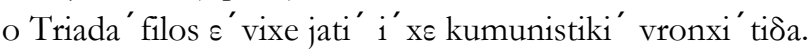


(a) « [...], Triadafyllos toussait car il avait une "bronchite communiste" - [...].» (La liqueur morte, p. 94)

On voit que le traducteur a choisi de mettre bronchite communiste entre guillemets pensant que le lecteur aura des difficultés à comprendre ce terme hors contexte. En effet, dans le contexte qui précède, nous apprenons que Triadafyllos est communiste. Un soir, en rentrant chez lui, à une époque de fortes tensions politiques, il découvre que deux policiers l'attendent dans son salon. Redoutant la suite, il réagit de façon nerveuse et se met à tousser. Nous interprétons cet énoncé en passant non pas par une loi - cas de l'emploi du connecteur $\varepsilon \pi \varepsilon \varepsilon \delta \dot{\eta}$ - mais par une règle (« si on est communiste et qu'on tousse, c'est qu'on a une bronchite communiste »). Cette observation pourrait nous amener à conclure que jaati se fonde principalement sur une règle alors que $\varepsilon \pi \varepsilon i \delta \eta^{\prime}$ sur une loi. Mais les données sont indéniablement plus compliquées.

Dans l'article de $2004 c$, nous avons affirmé que yrati peut être réservé à un raisonnement abductif, car ce connecteur est susceptible d'introduire le fait observé (l'effet) et non pas la cause. Nous avons alors pris comme point de départ la règle «quand on est malade [CAUSE], on a mauvaise mine [EFFET] » et nous avons fabriqué deux énoncés. En (19), le connecteur de subordination introduit la cause alors qu'en (20) yıazi est réservé à l'effet ${ }^{20}$ :

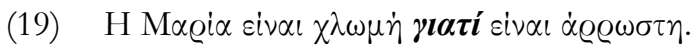
i Mari' a i' ne xlomi' jati' i' ne a' rosti.

(a) « Marie a mauvaise mine parce qu'elle est malade. »

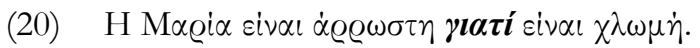
i Mari' a i' ne a' rosti jati' $i^{\prime}$ ne xlomi'.

(a) « Marie est malade car elle a mauvaise mine. »

Ainsi, l'exemple (20), qui par ailleurs est fortement modal, se base sur un raisonnement abductif : yıazi introduit le fait constaté ("avoir mauvaise mine ») et non pas la cause ("être malade »). Remarquons que $\varepsilon \pi \varepsilon \varepsilon \delta \dot{\eta}$ ne pourrait pas apparaittre dans un exemple comme le (20), car $\varepsilon \pi \varepsilon l \delta \dot{\eta}$ introduit uniquement la cause.

Passons à présent à un cas encore plus flagrant : la combinaison avec deux actes de parole dont l'un est un impératif

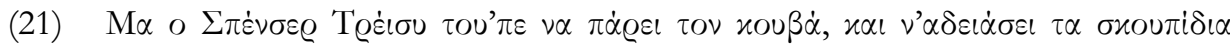

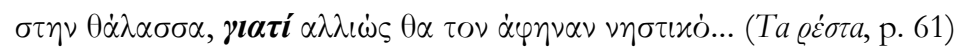

ma o Spenser Treisi tu pe na pa'ri ton kuva' ke n adja'si ta skupi' $\delta j a$ stin $\theta a^{\prime}$ lasa jati' aljo's $\theta$ a ton a' finan nistiko' ...

(a) «Mais Spencer Tracy lui dit d'attraper le seau et de vider les ordures dans la mer, s'il veut qu'on lui donne à manger... » (La petite monnaie, p. 73)

Litt. Mais Spenser Tracy lui a dit de prendre le seau et de vider les ordures à la mer, parce qu'autrement ils le laisseraient affamé...

${ }^{20}$ Babiniotis \& Clairis [2001 : 93, \$95] mentionnent ce caractéristique de yıati sans l'analyser. 
Essayons de formuler une règle, elle sera du type : «si tu ne vides pas les ordures dans la mer, on ne te donnera rien à manger ». Deux remarques s'imposent: d'une part, l'énoncé fait apparaitre une négation et d'autre part on voit que le connecteur ytat' introduit l'effet et non pas la cause. Nous rejoignons alors un résultat que nous avions établi précédemment [2004b, 2004c] à savoir que jıazi n'est pas toujours un connecteur de subordination (cf. également Mackridge, 1985/1990 : 381, \$8.4).

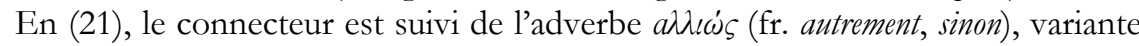
elliptique de la conjonction d'opposition $\varepsilon i \delta a ̈ \lambda \lambda \omega \varsigma$ / $\varepsilon \iota \delta a \lambda \lambda \iota \omega ́ s$ (fr. sinon). L'analyse étymologique de ce connecteur fait apparaitre une variante (grec ancien $\varepsilon l$, fr. $s i$ ) du connecteur hypothétique $\varepsilon \dot{a} v$ (fr. si). Parce qu'en (21) nous réunissons la présence de $\varepsilon a ́ v$ la règle sous-jacente « si tu ne vides pas les ordures dans la mer », nous pouvons dire que raazi connait un emploi biconditionnel [Moeschler, 1996: 228] consistant à combiner l'hypothèse et l'inférence. L'hypothèse et la négation de la règle sous-jacente portent sur la principale (étant à l’impératif) et non pas sur la subordonnée.

Jusqu'à présent, nous avons examiné deux constructions combinant deux actes de parole différents. Nous les résumons ici :

○ 《proposition interrogative $+\varepsilon \pi \varepsilon \iota \delta \dot{\eta}+$ subordonnée » (ex. 3) : la cause est présentée comme certaine; l'énonciateur cherche à établir un lien entre le contenu de la subordonnée et celui de la principale.

- "proposition impérative+ yıazi+subordonnée » (ex. 21 ou 22) : la structure est elliptique (seule la règle appliquée contient une négation).

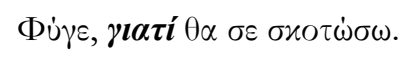

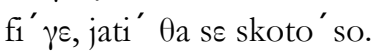

(a) «Pars, sinon je te tuerai. »

A cette liste, nous rajoutons également deux structures supplémentaires :

○ «proposition impérative+ $\varepsilon \pi \varepsilon l \delta \dot{\eta}+$ subordonnée » (ex. 23).

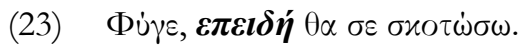

$\mathrm{fi}^{\prime} \gamma \varepsilon, \varepsilon p i \delta i^{\prime} \theta \mathrm{a} \varepsilon$ skoto' so.

(a) «Pars, parce que je te tuerai. »

Il existe indéniablement une différence entre les exemples (22) et (23) qui apparaît déjà dans leur traduction. La structure en (22) est elliptique (" pars, parce que si tu ne pars pas, je te tuerai ») et le connecteur yıati présente les conséquences possibles des actes du co-énonciateur (cf. ex. 21). Nous paraphrasons par «c'est uniquement parce que tu prendras la décision de ne pas partir que je te tuerai ». En revanche, (23) ne se prête pas à la même interprétation à cause de $\varepsilon \pi \varepsilon \delta \delta \dot{\eta}$. Il ne s'agit pas d'une menace au même titre qu'en (22) car on informe le co-énonciateur de ce qui va se produire inéluctablement s'il ne part pas. L'exemple est paraphrasé par "parce que je te tuerai, tu feras mieux de partir».

0 "proposition interrogative + plazi+subordonnée » comme dans

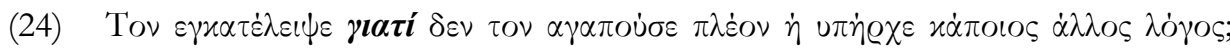

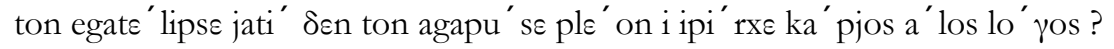


(a) «Elle l'a abandonné parce qu'elle ne l'aimait plus ou y avait-il une autre raison?»

Selon nous, le connecteur portant sur aimer et avoir une autre raison à la fois, introduit une cause plausible; elle est surtout présentée comme une parmi les causes susceptibles de provoquer le fait décrit dans la subordonnée. Hiérarchiquement, cette cause n'est pas placée très haut sur l'échelle argumentative [Ducrot, 1980 : 18].

Nous concluons que dans les structures que nous avons présentées les deux connecteurs ne se comportent pas de la même façon. Dans le cas d'une construction interrogative ou impérative avec $\varepsilon \pi \varepsilon \delta \delta \dot{n}$, on ne remet pas en question le certain de la subordonnée mais on cherche à établir le lien entre deux contenus propositionnels.

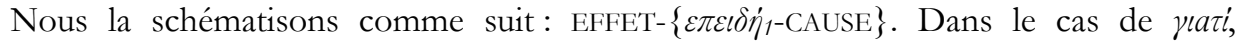
l'interrogation porte sur les deux contenus propositionnels: \{EFFET ylati-CAUSE\}. Mais yiazi connait un emploi supplémentaire que nous avons présenté en (22) ; quand il suit une proposition à l'impératif, il introduit l'opposition. Nous paraphrasons l'impératif par une proposition hypothétique faisant apparaittre une négation :

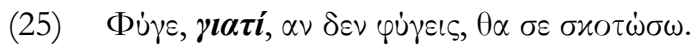

$\mathrm{fi}^{\prime} \gamma \varepsilon$, jati', an $\delta \varepsilon n \mathrm{fi}^{\prime} \gamma \mathrm{\gamma is}, \theta \mathrm{a} \varepsilon \varepsilon$ skoto' so.

(a) «Pars, parce que, si tu ne pars pas, je te tuerai.»

Nous proposons maintenant un exemple faisant apparaitre les deux connecteurs à la fois; il nous permettra de résumer certaines propriétés caractéristiques :

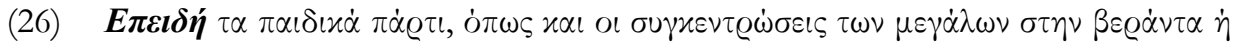

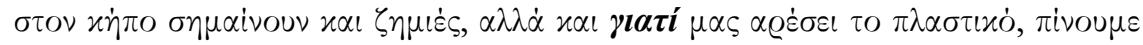

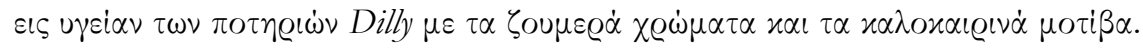
(Maison et décoration 38, p.30)

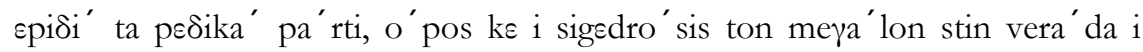
ston ki' po sime' nun ke zimie's, ala' ke jati' mas are' si to plastiko', pi' nume is i $\gamma i^{\prime}$ an ton potirio 'n Dili me ta zumera' xro' mata ke ta kalokerina' moti' va.

(a) «Parce que les fêtes d'enfants, comme les réceptions des adultes en terrasse ou dans le jardin se traduisent par des dégâts, mais aussi parce que nous aimons le plastique, nous buvons à la santé des verres Dilly avec leurs couleurs et motifs d'été. » (Maison et décoration 38, p. 30)

Le connecteur $\varepsilon \pi \varepsilon \delta \delta \dot{\eta}$ introduit une cause certaine. Si cette cause est un fait constaté à maintes reprises, nous pouvons généraliser; il introduit alors une vérité générale (phénomène similaire à l'antéposition du parce que). Le contenu de la

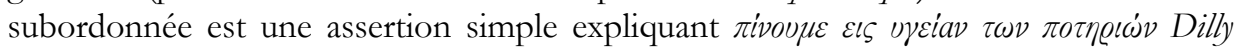
(fr. nous buvons à la santé des verres Dilly). De son côté, ylazi fait apparaitre un énonciateur se portant garant du contenu de la subordonnée et de la relation établie (modalité épistémique). Dans ce type de constructions, son pouvoir explicatif est très faible. 
Néanmoins, yıazi est susceptible d'introduire une cause certaine lorsqu'il fait appel à une loi (relation de portée ample), comme le montre l'exemple suivant :

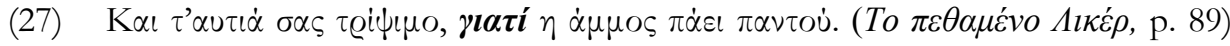
ket aftja' sas tri' psimo, jati' i a mos pa'i padu'.

(a) «Et frottez-vous bien les oreilles, car le sable entre partout... » (La liqueur morte, p. 113)

L'observation et l'analyse du connecteur yıazi soulèvent une série de questions sur son emploi et son interprétation sémantique. Pourquoi ce connecteur introduit-il une cause plausible mais dans quelques contextes une cause certaine ? Pourquoi a-t-on besoin d'un énonciateur qui s'engage, qui prend en charge la relation établie ? Il nous semble qu'il faudra chercher les réponses dans l'analyse étymologique du connecteur : elle fait apparaitre la préposition $\gamma ı$ (fr. pour), réservée pour exprimer le but, donc le non-réalisé en $\mathrm{T}_{0}$ (moment de l'énonciation). Cette notion de but est à l'origine de l'interprétation sémantique de la cause introduite par ce connecteur et de la position syntaxique de la subordonnée. Alors, yıazi introduit-il une cause ou une raison de dire ? Ce connecteur introduit une cause, le plus souvent plausible, mais nous avons également dégagé quelques facteurs permettant d'expliquer les cas où il introduit une cause certaine. En effet, lorsque les deux contenus propositionnels sont liés par une relation à la fois logique et chronologique, yrazi introduit une cause au sens propre du terme.

\section{A $\varphi o \dot{~: ~ l a ~ c a u s e ~ e t ~ l e ~ l o c u t e u r ~}$}

Cette dernière partie est consacrée au connecteur polysémique ạoú (fr. puisque, après que). Nous montrerons que, malgré ses similitudes avec $\varepsilon \pi \varepsilon \varepsilon \delta \dot{\eta}$, les deux connecteurs d'origine temporelle véhiculent une causalité différente.

Issu du grec ancien, a

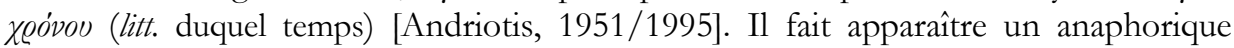
indiquant que la relation temporelle entre les deux contenus propositionnels est relative : on ne constate aucun indice explicite temporel (ex. adverbes) et le verbe de la subordonnée est au subjonctif lorsque la relation s'inscrit dans le futur. Sachant que le subjonctif grec est atemporel (on n'y trouve que des marques aspectuelles), l'anaphorique introduit par aøoú nous permet de définir la relation temporellement :

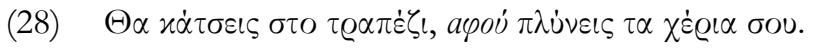

$\theta a$ ka 'tsis sto trape'zi, afu' pli' nis ta xje' rja su.

(a) «Tu te mettras à table après t'être lavé les mains. »

L'emploi causal étant dérivé de l'emploi temporel, la distinction entre les deux notions reste parfois extrêmement difficile. On a alors souvent recours aux temps de deux propositions pour distinguer l'emploi temporel de l'emploi causal. En (29), le connecteur marque à la fois la succession chronologique et logique de deux événements; chronologique car on est d'abord malade et puis on choisit de ne pas aller jouer, et logique car lorsqu'on est malade, on ne sort pas jouer : 


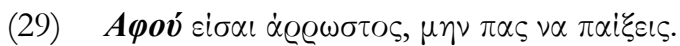
afu' i'se a' rostos, min pas na pe'ksis.

(a) «Puisque tu es malade, ne va pas jouer.»

Selon Triadafillidis [1941/1996: 397], la structure «a甲ov́+indicatif présent/ imparfait» introduit une subordonnée de cause, par opposition à la structure "a甲oú+indicatif aoriste/futur» qui présente une certaine ambiguité, car elle est susceptible d'exprimer soit la causalité, soit la temporalité :

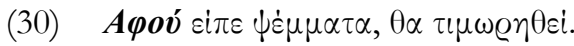
afu' i' pe pse' mata, $\theta$ a timori $\theta i^{\prime}$.

(a) «Puisqu'il a menti, il sera puni. »

Il faudrait introduire des facteurs supplémentaires pour désambiguïser la deuxième structure. Observons un couple d'exemples comme :

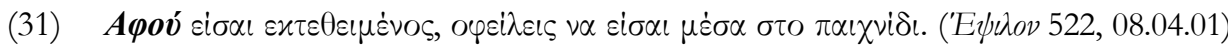

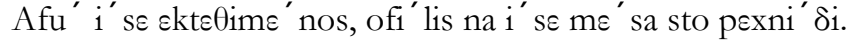

(a) «Puisque tu es exposé [devant le public], tu dois vraiment jouer ton rôle.» (Epsilon 522, 08.04.01)

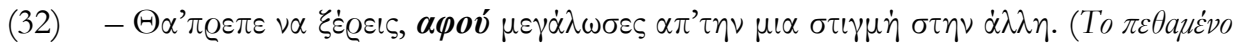
$\lambda(x \dot{e} \rho$, p. 130)

Oa prepe na kse' ris, afu' me a $^{\prime}$ loses ap tin mja stigmi' stin a'li.

(a) «-Tu devrais le savoir, puisque tu as grandi d'un jour à l'autre.» (La liqueur morte, p. 166)

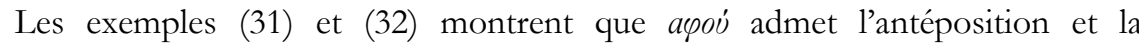
postposition respectivement tout comme $\varepsilon \pi \varepsilon i \delta \eta^{\prime}$ en (1) et (2). Le recours à la virgule signale deux segments qui, grâce à leur voisinage et le rapport logique existant entre eux, sont à relier. Mais, nous pouvons aussi constater une troisième construction faisant apparaitre un point final :

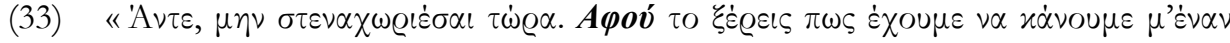

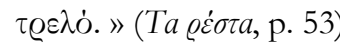

$\mathrm{a}^{\prime} \mathrm{d} \varepsilon$, min stenaxorje' se to' ra. Afu' to kse' ris pos $\varepsilon^{\prime}$ xume na ka' num $\mathrm{m}$ $\varepsilon^{\prime}$ nan trelo'.

(a) «Allez, ne t'en fais pas. Puisque tu le sais qu'on a affaire à un fou.» (La petite monnaie, p. 64)

Le recours au point final renvoie ainsi à une relation de dire où l'énonciateur justifie son choix d'énonciation. A l'oral, le point final est remplacé par la prosodie. Mais la différence entre les exemples (31) et (32) est plus que stylistique. En suivant l'analyse proposée par Kalokerinos [2001:321], nous constatons que la structure avec une virgule est un seul acte de parole provoquant chez le co-énonciateur le sentiment d'évidence ou de remise en question de ses compétences : 


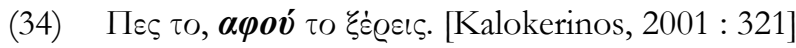
pes to, afu' to kse' ris.

(a) «Dis-le, puisque tu le sais.»

En revanche, la structure avec le point final encourage le co-énonciateur ou encore le console :

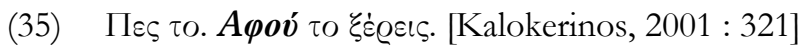
pes to. Afu' to kse' ris.

(a) «Dis-le. Puisque tu le sais.»

Tout comme l'exemple (33), l'exemple suivant fait apparaittre deux actes de parole dont l'un est une proposition interrogative :

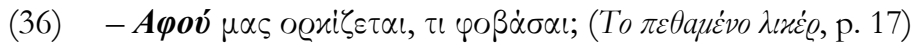
afu' mas orki' zetє, ti fova' se ?

(a) «-Puisqu'elle nous le jure, pourquoi as-tu peur? ? (La liqueur morte, p. 17)

La question ne porte guère sur le contenu de la subordonnée mais introduit plutôt l'étonnement de l'énonciateur. Jurer est présenté comme certain et on cherche à comprendre la raison de la peur du co-énonciateur.

Nous sommes amenée à conclure que le contenu de la subordonnée est présentée comme certain : le co-énonciateur peut ainsi vérifier ce contenu et le puiser soit dans un contexte plus large, soit dans un stock de connaissances partagées par la même communauté linguistique et/ou culturelle. Il en résulte que ạoú exprime une modalité aléthique. Ceci est également renforcé par le fait qu'on ne rencontre pas a dans un raisonnement paradoxal ; la relation est obligatoirement logique et étroitement liée au lexique comme le montre la série d'exemples qui suit:

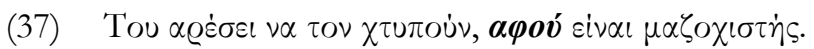
tu are'si na ton xtipu' $n$, afu' i' ne mazoxisti's.

(a) «Il aime qu'on le batte puisqu'il s'agit d'un masochiste. »

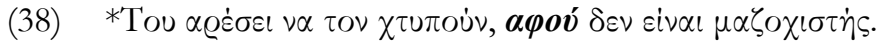
tu are'si na ton xtipu ' $n$, afu' $\delta \varepsilon n$ i' ne mazoxisti's.

(a) * «Il aime qu'on le batte puisqu'il ne s'agit pas d'un masochiste. »

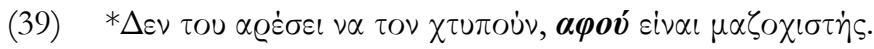
$\delta \varepsilon n$ tu are' si na ton xtipu' n, afu 'i ne mazoxisti's.

(a) * «Il n’aime pas qu'on le batte puisqu’il est masochiste. »

Dans tous les cas que nous avons cités, ạoú introduit une cause certaine même si elle relève du niveau discursif (ex. 35) - et établit une relation logique. Dans nos travaux antérieurs [2004c], nous avons montré que ce connecteur implique des raisonnements déductifs : prémisses et conclusions sont, toutes les deux, certaines. 
La notion de certitude caractérise alors a de la subordonnée. Ainsi, elle peut être au futur; ceci nous rapproche de l'emploi similaire de $\varepsilon \pi \varepsilon \varepsilon \delta \dot{\eta}$ dans l'exemple (5):

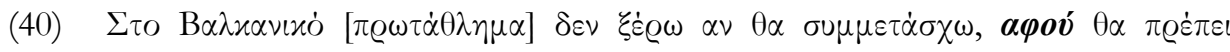

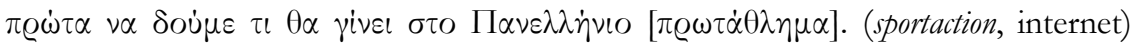
Sto valkaniko' [prota' $\theta$ lima] $\delta \varepsilon n$ kse' ro an $\theta$ a sumsta' sxo, afu' $\theta$ a pre' pi pro' ta na $\delta u^{\prime}$ me ti $\theta$ a $\gamma^{\prime}{ }^{\prime}$ ni sto Paneli 'nio [prota' $\theta$ lima].

(a) «Je ne sais pas si je participerai au championat [balkanique], puisque je devrai d'abord voir ce qui se passera avec le championat grec » (Sportaction, internet)

Si la notion de temps est à l'origine des connecteurs $\varepsilon \pi \varepsilon\llcorner\delta \dot{\eta}$ et $a \varphi \circ u$, s'ils connaissent tous les deux une position syntaxique libre et introduisent une cause certaine, peut-on les considérer comme synonymes ? La réponse est, d'après nous, négative, car - malgré ces éléments communs - ạoú opère à un niveau discursif et son contenu propositionnel n'est pas toujours indispensable pour la compréhension d'une relation. Il est donc donné entre paranthèses :

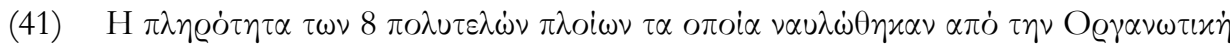

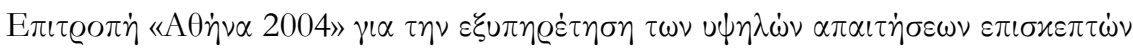

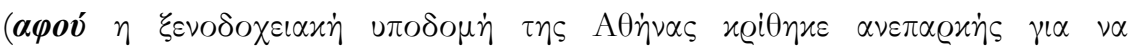

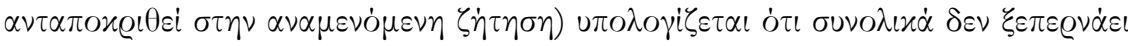

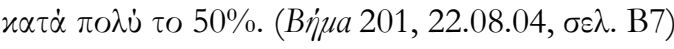

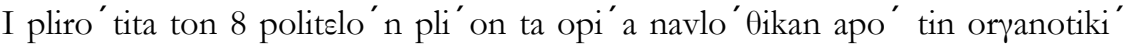

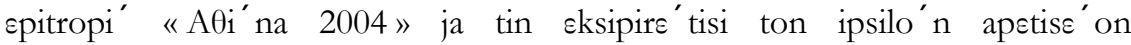

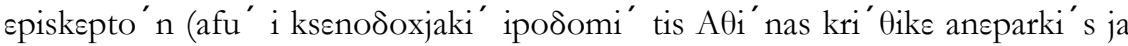

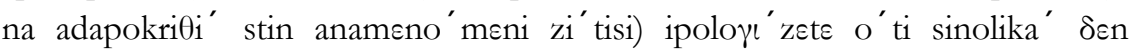
kseperna' $i$ kata' poli' to $50 \%$.

(a) «Les 8 bateaux de luxe qui ont été affrétés par le comité d'organisation Athènes 2004 pour répondre aux besoins des invités VIP (du fait que l'infranstructure hôtelière d'Athènes a été jugée insuffisante pour satisfaire la demande attendue) ne sont pleins qu'à moitié.» (Vima 201, 22.08.04, p. B7)

La présentation qui précède résume les emplois principaux ${ }^{21}$ de ce connecteur polysémique. Le trait distinctif entre son emploi temporel et son emploi causal réside non seulement dans les temps verbaux des deux propositions, mais également dans la construction syntaxique et la prosodie. En fait, la combinaison avec deux actes de parole différents nous invite à interpréter causalement la relation entre deux faits. D’autres linguistes, comme Kalokerinos [2001 : 319], proposent une analyse unifiée de ce connecteur qui combinerait deux stratégies discursives: l'énonciateur et le coénonciateur partagent le même stock de connaissances extra-linguistiques;

${ }^{21}$ Nous excluons de notre étude actuelle les constructions elliptiques et la notion de reprise. 
l'énonciateur ne prend pas la responsabilité du contenu de la subordonnée, mais il le présente comme l'opinion d'une tierce personne, souvent absente.

Ainsi, le champ de résonance n'est pas pris en charge par l'énonciateur dont le rôle se résume à un constat. Il est extérieur au fait donné dans la subordonnée et nous le considérons comme un locuteur au sens défini dans l'introduction de cet article. Nous pouvons, maintenant, expliquer l'agrammaticalité de l'exemple suivant :

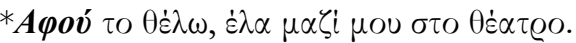

$$
\begin{aligned}
& \text { afu' to } \theta \varepsilon \text { 'lo, } \varepsilon^{\prime} \text { la mazi ' mu sto } \theta \varepsilon \text { 'atro. }
\end{aligned}
$$

(a) * «Puisque je le veux, viens avec moi au théâtre. »

Le locuteur n'observe plus un fait extérieur à lui mais il est directement impliqué non pas comme membre d'une communauté mais comme individu. En revanche, la première personne du pluriel où le locuteur fait partie d'un groupe, donne un énoncé sémantiquement correct :

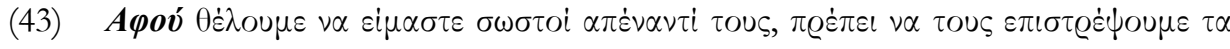
$\chi \varrho \dot{\eta} \mu \alpha \tau \alpha \pi O v \mu \alpha \varsigma \delta \dot{\alpha} \nu \varepsilon \iota \alpha \nu$.

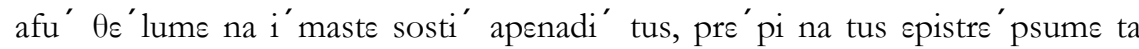
xri' mata pu mas da' nisan.

(a) «Puisque nous voulons être corrects avec eux, nous devons leur rendre l'argent qu'ils nous ont prêté. »

\section{Synthèse}

Nous espérons avoir démontré que les trois connecteurs causaux du grec moderne ne sont interchangeables ni sur le plan syntaxique ni sur le plan sémantique. Nous avons cherché à isoler leurs traits distinctifs en passant par le biais de la modalité ; cette dernière impliquant directement la notion de prise en charge et d'engagement de la part de l'énonciateur. Pour étudier les différents types de cause véhiculés par les trois connecteurs, nous nous sommes appuyée sur leur analyse étymologique. Nous avons ainsi pu constater que les connecteurs anciennement temporels à emploi causal en grec moderne (cf. $\varepsilon \pi \varepsilon \iota \delta \eta$ ) ou les connecteurs

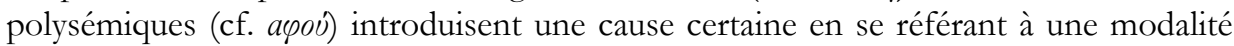
aléthique. En outre, $\varepsilon \pi \varepsilon i \delta \eta \dot{~ e t ~ a \varphi o v ́ ~ r e l e ̀ v e n t ~ a u s s i ~ d ' u n ~ m e ̂ m e ~ t y p e ~ d e ~ r a i s o n n e m e n t ~}$ déductif. Mais le niveau de prise en charge énonciative est différent. E $\pi \varepsilon \iota \delta \dot{\eta}$ fait apparaittre un énonciateur-garant du contenu de la subordonnée : nous avons à la fois

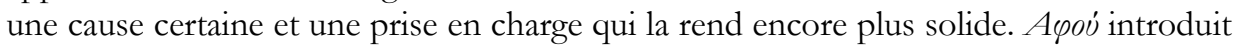
le même type de cause tout en faisant apparaître un locuteur. Ce dernier se présente comme un observateur des faits donnés dans la subordonnée.

D'autre part, il est apparu que yıati s'écarte des deux autres connecteurs causaux. A l'origine de cet écart, nous identifions la notion de but mise en évidence

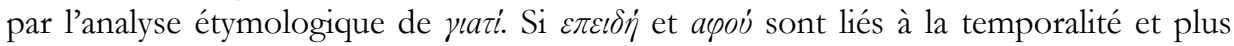
précisément à l'antériorité (fait attesté), la prise en charge énonciative du contenu propositionnel est «facultative ». En revanche, le but s'inscrivant dans un futur, il 
demande à être pris en charge par un énonciateur. Outre cette prise en charge indispensable, le but - lié à l'avenir - cache une incertitude. Cette incertitude se traduit par une cause plausible. Néanmoins, il ne faudrait pas laisser entendre que ytati n'introduit en aucun cas une cause certaine. Nous ne devons pas perdre de vue que rlazi est susceptible d'introduire une relation logique s'inscrivant souvent dans le passé, relation alors déjà réalisée et constatée. 


\section{RÉFÉRENCES BIBLIOGRAPHIQUES}

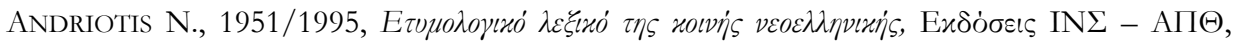
$\Theta \varepsilon \sigma \sigma \alpha \lambda o v i x \eta$.

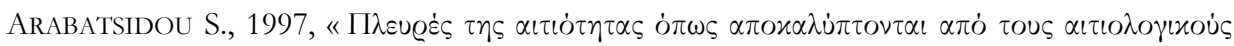

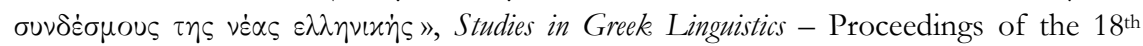
annual meeting of the Department of Linguistics, Aristotelian University of Thessaloniki, Editions Kyriakidis, Thessaloniki : 56-67.

Anscombre J.-C., 1984, «La représentation de la notion de cause en langue », Cabiers de Grammaire, Université de Toulouse-Le Mirail, 8 : 5-53.

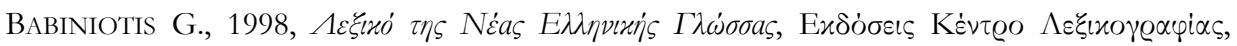
A $\theta \dot{\eta} \dot{\nu} \alpha$.

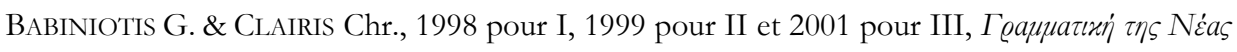

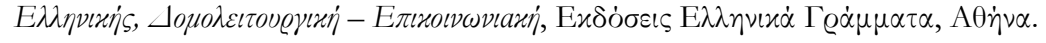

Borel M.-J., 1981, «Aspects logiques de l'explication», Travaux du Centre de Recherches Sémiologiques 38, Editions Université de Neuchâtel, Neuchâtel : 1-34.

Bybee J. \& Fleischman S., 1995, Modality in grammar and discourse, Editions Benjamins, Amsterdam / Philadelphia.

Culioli A., 1990, Pour une linguistique de l'énonciation - I, Editions Orphys, Paris.

DESCLÉS J.-P., 1998-1999, Notes du séminaire de D.E.A, Sorbonne-Paris IV.

Ducrot O., 1980, Les échelles argumentatives, Les éditions de Minuit, Paris.

—, 1984, Le Dire et le Dit, Les éditions de Minuit, Paris.

FORNEl De M., 1989, "Parce que et le problème de l'inférence", dans Cabiers de linguistique française 10, Editions Université de Genève, Genève, p. 171-192.

Galiotou E., 1999, Modélisation de la causalité à partir de l'analyse des phénomènes temporels dans les langues naturelles : application an grec moderne, Thèse de doctorat, Université Paris XII-Orsay, LIMSI-CNRS, Paris.

Groupe $\lambda-1$, 1975, «Car, parce que, puiseque», Revne romane, Copenhague, Copenhague Munkaard International Publishus, X-2 : 248-280.

HAMON S., 2005, La phrase double causale, thèse de doctorat, Université Paris X - Nanterre.

Holton D., MACKridge P., PhilipPAKi-WARburton I., 1997, Greek : a comprehensive grammar of the modern langage, Editions Routledge, London.

Hopper P.j \& TraugotT E.C., 1993, Grammaticalization, Editions Cambridge University Press, Cambridge.

JACKIEWICZ A., 2004, "Traces de l'énonciateur dans la causalité exprimée dans les textes », Intellectica 38, Publié avec le concours du CNRS et du Centre National du Livre : 43-68. 


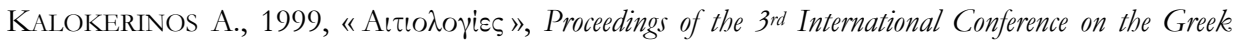
Language (1997), Editions E $\lambda \lambda \eta \nu \iota x \dot{\alpha} \Gamma \varrho \dot{\alpha} \mu \mu \alpha \tau \alpha$, Athens : 141-149.

—, 2001, «A A University Studio Press, Thessaloniki : 315-322.

—, 2004, "The justifying connection mostly in Greek », Journal of Greek Linguistics 5, Editions Benjamins, Amsterdam : 27-80.

KALOKERINOS A. \& FrASER B., non publié, «Implicative discoursive markers: a comparative inquiry into Greek and English », paper presented as a planary lecture in the $2^{\text {nd }}(2001)$ International Conference In Contrastive Semantis, Newnham College, Cambridge University.

KORTMANN B., 1996, Adverbial subordination: a typology and bistory of adverbial subordinators based on European Langages, Editions Mouton de Gruyter, Berlin.

KouTOUPI-KITIS E., 1995, «Some preliminary remarks on the greek causative conjonctions

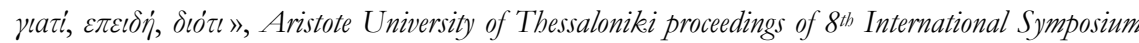
on English and Greek : description and/or comparison of the two langages (1994), Editions Kakouriotis, Thessaloniki : 306-318.

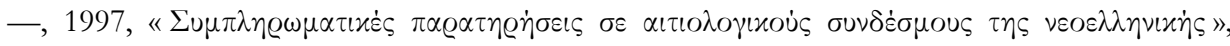
Studies in Greek Linguistics - Proceedings of the 17th annual meetings of the Department of Linguistics (1996), Aristotelian University of Thessaloniki, Editions Kyriakidis, Thessaloniki, p. 424-437.

—, 2000a, "Temporality and causality: The case of Greek subordinating connectives ", Revue de sémantique et pragmatique 8, Editions Presses Universitaires d’Orléans, Orléans : 121-141.

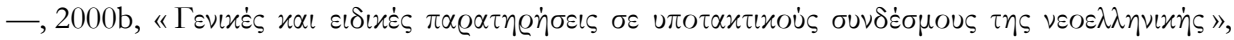
Studies in Greek Linguistics - Proceedings of the 20th annual meetings of the Department of Linguistics (1999), Aristotelian University of Thessaloniki, Editions Kyriakidis, Thessaloniki : 222-233.

—, à paraitre, « Causal connectives: the evidence from Greek », ICLA 1997 Conference, Amsterdam.

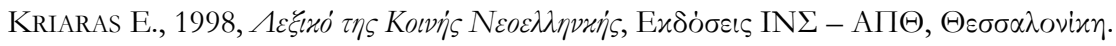

LidDELl H. \& G. SCOTT R., 1968, A Greek-English Lexicon, Editions Oxford University, Oxford.

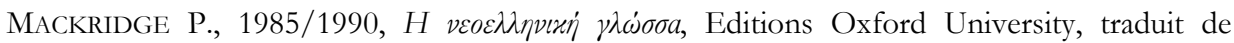
l'anglais par Petropoulos, Editions Patakis, Athènes.

MARTIN R., 1973, «Le mot puisque: notion d'adverbe de phrase et de présupposition sémantique ", Studia Neophilologica, XLV : 104-114.

MOesCHLER J., 1986, «Connecteurs pragmatiques, lois de discours et stratégies interprétatives : parce que et la justification énonciative ", dans Cahiers de linguistique française 7, Éditions Université de Genève, Genève : 149-168.

SPERBER D. \& WiLSON D., 1986/1989, La pertinence, traduit de l'anglais par A. Gerschenfeld et D. Sperber, Editions De Minuit, Paris. 
SWEETSER E., 1990, From etymology to pragmatics, metaphorical and cultural aspects of semantic structure, Editions Cambridge University Press, Great Britain.

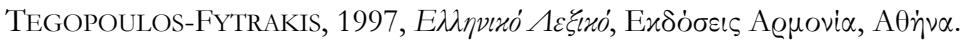

TORCK D., 1995, Aspects de la causalité discursive en français oral contemporain, Editions Studies in language and language use, Utigate IFOTT, Amsterdam.

TRAugOtT E \& KÖNIng E, 1991, "The semantics-pragmatics of grammaticalization revisited », in Traugott E. \& Heine B., eds., Approaches to grammaticalization v.II, Editions Benjamins, Amsterdam : 189-218, v.I.

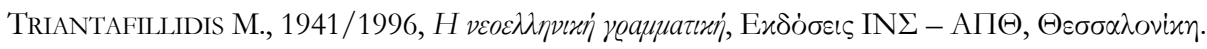

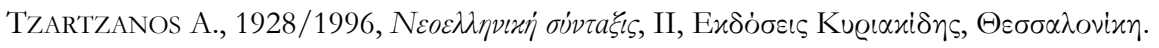

VAlma E., 2005, «Parce que et ses équivalents fonctionnels en grec moderne : $\varepsilon \pi \varepsilon \varepsilon \delta \eta \dot{~ e t ~} \gamma \iota a \tau i »$, dans Langues : histoires et usages dans l'aire méditarranéenne (à l'issue du colloque international La Méditerranée et ses langues, 2002), Editeur T. Arnavielle, Editions L'Harmattan, Paris : 89-100.

—, 2004a, L'expression de la causalité en français et en grec moderne - étude contrastive, Thèse non publiée, Université Paris VII - Denis Diderot, Paris.

—, 2004b, " Tiati et ses équivalents fonctionnels en français», dans les actes du colloque international (2002) Traduire an XXIe siècle: tendances et perspectives, Editions University Studio Press, Thessalonique : 556-563.

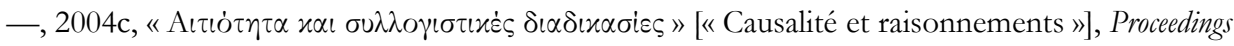
of the $6^{\text {th }}$ International Conference on Greek linguistics, ebook/ISBN 960-88268-0-2, University of Rethymno, Creta.

—, 1999, L'expression de la causalité en français et en grec moderne, mémoire de DEA, Université Paris VII - Denis Diderot, Paris.

VION R., 2001, «Modalités, modalisations et activités langagières », Marges Linguistiques 2, version électronique.

\section{SOURCES DU CORPUS}

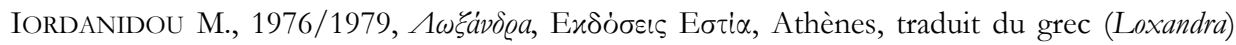
par B.Molfessis, Editions Institut Français d'Athènes - Actes Sud, Athènes / Paris.

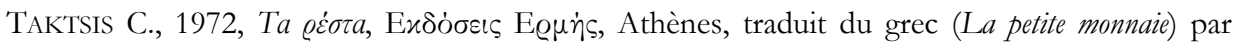
M.Volkovitch, 1988, Editions Gallimard, Paris.

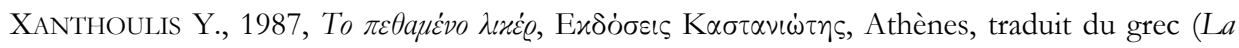
Liqueur Morte) par L.Rotis, 1991, Editions Hatier, Paris. 